\title{
Brill-Noether general K3 surfaces with the maximal number of elliptic pencils of minimal degree
}

\section{Michael Hoff ${ }^{1}$ iD $\cdot$ Andreas Leopold Knutsen ${ }^{2}$}

Received: 15 May 2020 / Accepted: 24 August 2020 / Published online: 4 September 2020

(c) The Author(s) 2020

\begin{abstract}
We explicitly construct Brill-Noether general $K 3$ surfaces of genus 4, 6 and 8 having the maximal number of elliptic pencils of degrees 3, 4 and 5, respectively, and study their moduli spaces and moduli maps to the moduli space of curves. As an application we prove the existence of Brill-Noether general $K 3$ surfaces of genus 4 and 6 without stable LazarsfeldMukai bundles of minimal $c_{2}$.
\end{abstract}

Keywords K3 surfaces · Unirationality · Moduli map · Lazarsfeld-Mukai bundle

Mathematics Subject Classification (2010) $14 \mathrm{~J} 28 \cdot 51 \mathrm{M} 15 \cdot 14 \mathrm{Q} 10 \cdot 14 \mathrm{~J} 10$

\section{Introduction}

It is well-known that a general curve of genus $g \leq 9$ or $g=11$ can be realized as a linear section of a primitively polarized $K 3$ surface, cf. [26,28]. Since for even $g$ a general curve $C$ carries a finite number of pencils of minimal degree $\frac{g}{2}+1$, it is natural to ask whether one can simultaneously extend $C$ and all or some of these pencils to some $K 3$ surfaces for $g=4,6,8$. This question is connected to the existence of non-stable Lazarsfeld-Mukai bundles. Indeed, the Lazarsfeld-Mukai bundle associated to a pencil on a smooth curve on the $K 3$ surface induced by an elliptic pencil on the surface is necessarily not stable, cf. Lemma 5.1.

Using vector bundle methods, Mukai [29] showed that the projective model of any BrillNoether general $K 3$ surface $(S, L)$ is obtained as sections of homogeneous varieties for $g \in\{6, \ldots, 10,12\}$. By definition, cf. [29, Def. 3.8], a polarized $K 3$ surface $(S, L)$ of genus $g$ is Brill-Noether general if $h^{0}(M) h^{0}(N)<g+1=h^{0}(L)$ for any non-trivial decomposition $L \sim M+N$. In these low genera this is equivalent to all the smooth curves in the linear system $|L|$ being Brill-Noether general, due to techniques in [13,22] (see [14, Lemma 1.7]).

$凶 \quad$ Michael Hoff

hahn@math.uni-sb.de

Andreas Leopold Knutsen

andreas.knutsen@math.uib.no

1 Universität des Saarlandes, Campus E2 4, 66123 Saarbrücken, Germany

2 Department of Mathematics, University of Bergen, 5020 Bergen, Norway 
Using Mukai's results, we will study projective models of Brill-Noether general $K 3$ surfaces of genus $g \in\{4,6,8\}$ containing the maximal possible number of elliptic pencils of degree $\frac{g}{2}+1$.

The goal of our paper is threefold:

(1) We provide explicit constructions/equations of $K 3$ surfaces with special geometric features.

(2) We describe their moduli spaces as lattice polarized $K 3$ surfaces and the corresponding moduli map to the moduli space of curves of genus $g$.

(3) We study the slope-stability of Lazarsfeld-Mukai bundles of hyperplane sections on such K3 surfaces.

Our main results are the following.

- Section 3: We prove that a general curve $C$ of genus 4 is a linear section of a smooth $K 3$ surface $S$ such that its two $g_{3}^{1}$ s (which are well-known to be auto-residual) are induced by two elliptic pencils $\left|E_{1}\right|$ and $\left|E_{2}\right|$ on $S$ satisfying $C \sim E_{1}+E_{2}$, cf. Proposition 3.4. Furthermore, the moduli space parametrizing such $K 3$ surfaces is unirational (and 18-dimensional), cf. Proposition 3.2. We believe that these results should be known, but could not find any reference.

- Section 4: A general curve $C$ of genus 6 carries precisely five pencils $\left|A_{1}\right|, \ldots,\left|A_{5}\right|$ of minimal degree 4 which satisfy $2 K_{C} \sim A_{1}+\cdots+A_{5}$ (see [4, p. 209ff]). We prove that $C$ is a linear section of a smooth $K 3$ surface $S$ such that its five $g_{4}^{1}$ s are induced by five elliptic pencils $\left|E_{1}\right|, \ldots,\left|E_{5}\right|$ on $S$ satisfying $2 C \sim E_{1}+\cdots+E_{5}$, cf Theorem 4.3(a). We prove that the moduli space parametrizing such pairs $(S, C)$ is unirational, cf. Theorem 4.3(b). The moduli space of the underlying $K 3$ surfaces was already studied in [5] where it was shown to be birational to the moduli space $\mathcal{M}_{6}$ of curves of genus 6 (and therefore, rational, cf. [34]). Our approach shows that this moduli space is exactly the locus of Brill-Noether general $K 3$ surfaces that cannot be realized as quadratic sections of a smooth quintic Del Pezzo threefold (but as quadratic sections of a cone over a smooth quintic Del Pezzo surface), cf. Remark 4.4(b).

- Section 6: A general curve $C$ of genus 8 carries precisely 14 pencils of degree 5 . An easy lattice computation shows that at most 9 can be extended to a $K 3$ surface containing $C$. We prove that this bound is reached in codimension 3 in the moduli space $\mathcal{M}_{8}$, and for a general curve only six out of its 14 pencils can be extended to elliptic pencils on a $K 3$ surface, cf. Corollary 6.11 . We prove that the moduli spaces of such $K 3$ surfaces containing $i$ elliptic pencils are unirational for $1 \leq i \leq 6$ and $i=9$, cf. Theorems 6.7 and 6.8 .

- Section 5: The $K 3$ surfaces constructed in Sect. 3 (respectively 4) provide examples of $K 3$ surfaces without stable (resp. semistable) Lazarsfeld-Mukai bundles with $c_{2}=3$ (resp. 4), cf. Corollary 5.2 (resp. 5.3). This shows in particular the sharpness of a result of Lelli-Chiesa [23, Thm. 4.3], cf. Remark 5.4.

\section{Notation and conventions}

We work over $\mathbb{C}$. We will denote $V_{n}$ an $n$-dimensional vector space and $G\left(k, V_{n}\right)$ (respectively $G\left(V_{n}, k\right)$ ) the Grassmannian of $k$-dimensional sub- (resp. quotient-) spaces of $V_{n}$. The projective space of one-dimensional sub- (resp. quotient-) spaces is denoted $\mathbb{P}_{*}\left(V_{n}\right)$ (resp. $\left.\mathbb{P}^{*}\left(V_{n}\right)\right)$. 


\section{Lattice polarized $K 3$ surfaces and their moduli spaces}

Let $\mathfrak{h}$ be a lattice. The moduli space $\mathcal{F}^{\mathfrak{h}}$ of $\mathfrak{h}$-polarized $K 3$ surfaces parametrizes pairs $(S, \varphi)$ (up to isomorphism) consisting of a $K 3$ surface $S$ and a primitive lattice embedding $\varphi: \mathfrak{h} \rightarrow \operatorname{Pic}(S)$ such that $\varphi(\mathfrak{h})$ contains an ample class. It is a quasi-projective irreducible $(20-\operatorname{rk}(\mathfrak{h}))$-dimensional variety by [11].

If $(S, \varphi) \in \mathcal{F}^{\mathfrak{h}}$ is an $\mathfrak{h}$-polarized $K 3$ surface and $L \in \mathfrak{h} \cong \varphi(\mathfrak{h})$ is a distinguished class with $L^{2}=2 g-2 \geq 2$, one may consider the open subset

$$
\mathcal{F}_{g}^{\mathfrak{h}}=\left\{(S, \varphi) \mid(S, \varphi) \in \mathcal{F}^{\mathfrak{h}} \text { and } L \text { ample }\right\}
$$

of the moduli space $\mathcal{F}^{\mathfrak{h}}$, which may also be considered as a subset of the moduli space $\mathcal{F}_{g}$ of polarized $K 3$ surfaces of genus $g$. Furthermore, let $\mathcal{P}_{g}^{\mathfrak{h}}$ denote the moduli space of triples $(S, \varphi, C)$ where $C \in|L|$ is a smooth irreducible curve in the distinguished linear system. Then we have moduli maps

$$
m_{g}: \mathcal{P}_{g}^{\mathfrak{h}} \rightarrow \mathcal{M}_{g}
$$

Since in our cases of study it will be clear what the distinguished class $L$ will be, we will often skip the index $g$ in $\mathcal{F}_{g}^{\mathfrak{h}}$ and $\mathcal{P}_{g}^{\mathfrak{h}}$.

\section{$3 K 3$ surfaces of genus 4}

We will show the unirationality of the moduli space $\mathcal{F}^{\mathfrak{U}(3)}$ of lattice polarized $K 3$ surfaces where $\mathfrak{U}$ is the hyperbolic lattice of rank 2 . We believe that this result should be well-known, but we could not find any reference.

The following example is well-known, but we include it for the sake of the reader and it serves as an introduction for our next results and constructions.

Example 3.1 (The moduli space of $K 3$ surfaces of genus 4) A smooth polarized $K 3$ surface $S \subset \mathbb{P}^{4}$ of genus 4 is the complete intersection of a quadric $Q$ and a cubic hypersurface $Y$ in $\mathbb{P}^{4}$. The quadric $Q=V(q)$ and the cubic $Y=V(y)$ are given by polynomials $q \in$ $H^{0}\left(\mathbb{P}^{4}, \mathcal{O}_{\mathbb{P}^{4}}(2)\right)$ and $y \in H^{0}\left(\mathbb{P}^{4}, \mathcal{O}_{\mathbb{P}^{4}}(3)\right)$ of degrees 2 and 3 , respectively.

The moduli space $\mathcal{F}_{4}$ of $K 3$ surfaces of genus 4 is described as follows. The quadric has to be of rank at least 4 since otherwise $S$ will be singular. Let $V \subset H^{0}\left(\mathbb{P}^{4}, \mathcal{O}_{\mathbb{P}^{4}}(2)\right)$ be the open subset consisting of quadratic equations of rank $\geq 4$. For a chosen equation $q$ we need to pick a cubic $y$ such that $y$ is no multiple of $q$, and the intersection of $Q$ and $Y$ should be smooth. Let $V_{q}$ be the five-codimensional quotient of $H^{0}\left(\mathbb{P}^{4}, \mathcal{O}_{\mathbb{P}^{4}}(3)\right)$ parametrizing non-multiples of $q$. The desired cubic equations are parametrized by an open subset $W_{q} \subset V_{q}$. Let $W$ be the iterated Grassmannian

$$
W \stackrel{G\left(1, W_{q}\right)}{\longrightarrow} \mathbb{P}_{*}(V) \cong \mathbb{P}^{14}
$$

whose fibers are Grassmannians of one-dimensional subspaces of $W_{q}$. Then $\mathcal{F}_{4}$ is birational to $W$ modulo the automorphism group of $\mathbb{P}^{4}$ and therefore $\mathcal{F}_{4}$ is unirational. Note further that a dimension count yields

$$
\operatorname{dim} V+\operatorname{dim} W_{q}-\operatorname{dim} P G L(5)=\left(\left(\begin{array}{l}
6 \\
2
\end{array}\right)-1\right)+\left(\left(\begin{array}{l}
7 \\
3
\end{array}\right)-1-5\right)-\left(5^{2}-1\right)=19,
$$

as expected. 


\subsection{K3 surfaces of genus 4 with an elliptic pencil of degree 3}

With notation as in the previous example let $S \subset \mathbb{P}^{4}$ be a smooth $K 3$ surface of genus 4 with polarization $L=\mathcal{O}_{S}(1)$. Assume that there exists a class $E \in \operatorname{Pic}(S)$ such that $E^{2}=0$ and $E . L=3$. By Riemann-Roch, $h^{0}(S, E)=2$ and $E^{\prime}$ is a smooth elliptic normal curve for general $E^{\prime} \in|E|$. Hence we get a pencil of elliptic normal curves. The pencil induces a rational normal scroll

$$
X=\bigcup_{E^{\prime} \in|E|} \overline{E^{\prime}} \subset \mathbb{P}^{4}
$$

of dimension 3 and degree 2 where $\overline{E^{\prime}}=\mathbb{P}^{2}$ is the linear span of $E^{\prime}$. Thus the scroll $X$ is the unique quadric hypersurface containing $S$. Furthermore, the scroll $X$ is singular in a point (since any two different projective planes in $\mathbb{P}^{4}$ intersect and $X$ cannot be singular along a line), that is, $X$ is a rank 4 quadric.

We remark that the residual class $L-E$ is a second elliptic pencil of degree 3 on $S$ and the maximal number of such pencils is two since $S \subset \mathbb{P}^{4}$ is generated by a unique quadric. We get a $K 3$ surface whose Picard lattice contains the intersection matrix with respect to the ordered basis $\{L, E\}$ (respectively $\{L-E, E\}$ )

$$
\left(\begin{array}{ll}
6 & 3 \\
3 & 0
\end{array}\right)\left(\operatorname{resp} .\left(\begin{array}{ll}
0 & 3 \\
3 & 0
\end{array}\right)=\mathfrak{U}(3)\right)
$$

where $\mathfrak{U}$ is the hyperbolic lattice of rank 2 and $L$ is the sum of the two basis elements of square 0 . In general $\operatorname{Pic}(S) \cong \mathfrak{U}(3)$ ( $\operatorname{such} K 3$ surfaces exist by [24, Thm. 2.9(i)] or [31]), in which case $L$ is the unique element (up to sign) of square 6 , hence genus 4 , which is easily seen to be very ample by the classical results of Saint-Donat [32]. Furthermore, such a $K 3$ surface $(S, L)$ is Brill-Noether general.

Recall from the introduction that $\mathcal{F}^{\mathfrak{U}(3)}$ is the moduli space of $\mathfrak{U}(3)$-polarized $K 3$ surfaces.

Proposition 3.2 The moduli space $\mathcal{F}^{\mathfrak{U}(3)}$ is unirational.

Proof By what we said, a general element in $\mathcal{F}^{\mathfrak{U}(3)}$ comes equipped with a unique embedding into $\mathbb{P}^{4}$ (up to the action of the projective linear group), as a complete intersection of a cubic and a rank 4 quadric, singular in a point. The converse holds true: if a smooth surface $S \subset \mathbb{P}^{4}$ is a complete intersection of a rank 4 quadric hypersurface $Q$ and a cubic hypersurface, then the two rulings on $Q$ cut out two residual elliptic pencils of degree 3 on $S$.

We describe a birational model of the moduli space $\mathcal{F}^{\mathfrak{U}(3)}$ by modifying the construction in Example 3.1, keeping the notation therein.

Let $V^{\prime} \subset H^{0}\left(\mathbb{P}^{4}, \mathcal{O}_{\mathbb{P}^{4}}(2)\right)$ be the subset of quadratic equations of rank 4 . Since a rank 4 quadric is a cone over a smooth quadric in $\mathbb{P}^{3}$, the space $V^{\prime}$ is isomorphic to an open subset of a $\mathbb{P}^{4}$-bundle over $\mathbb{P} H^{0}\left(\mathbb{P}^{3}, \mathcal{O}_{\mathbb{P}^{3}}(2)\right)$ and is therefore unirational. Pick $q \in V^{\prime}$. Then the moduli space $\mathcal{F}^{\mathfrak{U}(3)}$ is birational to the iterated Grassmannian

$$
W^{\prime} \stackrel{G\left(1, W_{q}\right)}{\longrightarrow} V^{\prime}
$$

modulo automorphisms and is therefore unirational, too. (Since $\operatorname{dim} V^{\prime}=\left(\begin{array}{l}5 \\ 2\end{array}\right)-1+4=13$, a dimension count yields that $\mathcal{F}^{\mathfrak{U}(3)}$ is a codimension one subspace of $\mathcal{F}_{4}$, as expected.)

Remark 3.3 Let $\mathfrak{U}$ be the hyperbolic lattice of rank 2. Even if the example above should be classically known, we only found in the literature unirationality results of $\mathcal{F}^{\mathfrak{U}(n)}$ for $n=1$ and 
2 (cf. [9]). Elliptic surfaces are parametrized by $\mathcal{F}^{\mathfrak{U}}$ and double covers of $\mathbb{P}^{1} \times \mathbb{P}^{1}$ branched along a curve of bidegree $(4,4)$ are parametrized by $\mathcal{F}^{\mathfrak{U}(2)}$.

Recall from the introduction that $\mathcal{P}^{\mathfrak{U}(3)}$ is the moduli space of triples $(S, \varphi, C)$ where $(S, \varphi) \in \mathcal{F}^{\mathfrak{U}(3)}$ and $C \in|L|$ is a smooth curve of genus 4 in the distinguished linear system. Also recall that a general curve of genus 4 has exactly two distinct $g_{3}^{1} \mathrm{~s}$, which are autoresidual.

Proposition 3.4 The moduli map $\mathcal{P}^{\mathfrak{U}(3)} \rightarrow \mathcal{M}_{4}$ is dominant. In particular, a general curve $C$ of genus 4 is a linear section of a smooth $K 3$ surface $S$ such that its two $g_{3}^{1}$ s are induced by two elliptic pencils $\left|E_{1}\right|$ and $\left|E_{2}\right|$ on $S$ satisfying $C \sim E_{1}+E_{2}$.

Proof We consider a general curve $C \subset \mathbb{P}^{3}$ of genus 4 , canonically embedded into $\mathbb{P}^{3}$, which is a complete intersection of a smooth quadric $Q^{\prime}$ and a cubic $Y^{\prime}$ (the quadric $Q^{\prime}$ is smooth since the two $g_{3}^{1}$ s are distinct). We will construct a $K 3$ surface $S \in \mathcal{F}^{\mathfrak{U}(3)}$ with the curve $C$ as a linear section. Therefore, we choose a $\mathbb{P}^{4}$ containing the ambient space $\mathbb{P}^{3}$ of the curve. Let $Q \subset \mathbb{P}^{4}$ be a cone over the quadric $Q^{\prime} \subset \mathbb{P}^{3}$, that is, a rank 4 quadric whose hyperplane section with the given $\mathbb{P}^{3}$ is $Q^{\prime}$. Let $Y \subset \mathbb{P}^{4}$ be any cubic hypersurface such that $Y \cap \mathbb{P}^{3}=Y^{\prime}$. The surface $S \subset \mathbb{P}^{4}$ can be chosen as the complete intersection of $Q$ and $Y$. Then, the pair $(S, C)$ is an element of $\mathcal{P}^{\mathfrak{U}(3)}$ by construction, and the dominance of the moduli map follows. The last statement is immediate.

Remark 3.5 Similarly in [21] it is shown that the moduli space of $K 3$ surfaces admitting a special automorphism of order 3 is birational to the moduli space of curves of genus 4 (see also [6] for its generalization).

\section{K3 surfaces of genus 6}

Inspired by the seminal work of Mukai [27], we will construct a Brill-Noether general $K 3$ surface $S$ of genus 6 where every complete pencil of degree 4 on a hyperplane section of $S$ is induced by an elliptic pencil on $S$. Furthermore, we show that the moduli space of such lattice polarized $K 3$ surfaces is unirational.

We briefly recall Mukai's construction. Let $(S, L)$ be a Brill-Noether general $K 3$ surface of genus 6. There exists a unique stable (rigid) vector bundle $\mathcal{E}$ of rank 2 on $S$ with $c_{1}(\mathcal{E})=L$, $h^{0}(S, \mathcal{E})=5$ and $h^{i}(S, \mathcal{E})=0$ for $i=1,2$ [16, Prop. 5.2.7]. This bundle induces an embedding of $S$ into the Grassmannian $G\left(V_{5}, 2\right)$, where $V_{5}=H^{0}(S, \mathcal{E})$, by sending $s \in S$ to the fiber $\mathcal{E}_{s}=\mathcal{E} \otimes \mathcal{O}_{s}$. As described in [27], a Brill-Noether general $K 3$ surface $S$ is the intersection of a linear section of codimension 3 (or 4) and a quadratic section of either the Plücker embedding $G\left(V_{5}, 2\right) \subset \mathbb{P}^{9}$ or of its cone $\widehat{G\left(V_{5}, 2\right)} \subset \mathbb{P}^{10}$, respectively.

In order to get an elliptic pencil of degree 4 on a $K 3$ surface, we need special sections of the following form. If the linear section of codimension 3 cuts a sub-Grassmannian of type $G(4,2)$ in a quadric surface, we get an elliptic normal curve of degree 4 on $S$ as the intersection of this quadric surface with the quadric section. A pencil of Grassmannians of type $G(4,2)$ induces a pencil of elliptic curves on $S$ and can be controlled in the dual space in the following way.

Lemma 4.1 A hyperplane corresponds to a point in the dual Grassmannian $G\left(2, V_{5}\right) \subset \mathbb{P}^{9 \vee}$ if and only if it cuts out a Schubert subvariety. Moreover, the Schubert variety is a onedimensional union of Grassmannians of type $G(4,2)$ contained in $G\left(V_{5}, 2\right)$. 
We will prove the same statement for the Grassmannian $G\left(V_{6}, 2\right)$ in the next section (cf. Sect. 6.1.1) and leave this proof to the readers. Note that two Grassmannians of type $G(4,2)$ in $G\left(V_{5}, 2\right)$ intersect in a 2-plane. Hence, two elliptic curves of distinct pencils of degree 4 with respect to $L$ intersect in two points. This can also be seen in the following way: if $E_{1}$ and $E_{2}$ are such elliptic curves, then $E_{1} . E_{2} \geq 2$ (as each $\left|E_{i}\right|$ is a pencil); moreover, since $\left(L-E_{1}\right)^{2}=2$, one also has $4-E_{1} \cdot E_{2}=E_{2} \cdot\left(L-E_{1}\right) \geq 2$, whence $E_{1}$. $E_{2} \leq 2$. Also inspired by the previous example of $K 3$ surfaces of genus 4 , we will construct a $K 3$ surface with Picard lattice of the following form:

$$
\left(\begin{array}{ccccc}
10 & 4 & 4 & \ldots & 4 \\
4 & 0 & 2 & \ldots & 2 \\
4 & 2 & 0 & \ddots & \vdots \\
\vdots & \vdots & \ddots & \ddots & 2 \\
4 & 2 & \ldots & 2 & 0
\end{array}\right)
$$

An easy computation shows that the rank can be at most five (otherwise the matrix has at least two non-negative eigenvalues). Let $\mathfrak{M}$ be the lattice given by the following intersection matrix

$$
\mathfrak{M}=\left(\begin{array}{ccccc}
10 & 4 & 4 & 4 & 4 \\
4 & 0 & 2 & 2 & 2 \\
4 & 2 & 0 & 2 & 2 \\
4 & 2 & 2 & 0 & 2 \\
4 & 2 & 2 & 2 & 0
\end{array}\right)
$$

We denote $S$ a $K 3$ surface with the above Picard lattice $\mathfrak{M}$ of rank 5 (which exists by [24, Thm. 2.9(i)] or [31]) and let $L$ be the basis element of square 10 . Let $E_{i}, i=1, \ldots, 4$, be the generators of square zero. Note that $E_{5}:=2 L-E_{1}-E_{2}-E_{3}-E_{4}$ is also an element of square zero and degree 4 with respect to $L$.

The lattice $\mathfrak{M}$ is also generated by elements $s_{0}, s_{1}, \ldots, s_{4}$ where $s_{0}=E_{1}+\cdots+E_{4}-L$ and $s_{i}=s_{0}-E_{i}, i=1, \ldots, 4$, with intersection matrix

$$
\left(\begin{array}{ccccc}
2 & 0 & 0 & 0 & 0 \\
0 & -2 & 0 & 0 & 0 \\
0 & 0 & -2 & 0 & 0 \\
0 & 0 & 0 & -2 & 0 \\
0 & 0 & 0 & 0 & -2
\end{array}\right) .
$$

(This is the lattice considered in [5].) We may assume that $s_{0}$ is big and nef by standard arguments (see [7, VIII, Prop. 3.10]). Note that $L=3 s_{0}-\sum_{i=1}^{4} s_{i}, E_{i}=s_{0}-s_{i}$ for $i=1, \ldots, 4$ and $E_{5}=6 s_{0}-3 \sum_{i=1}^{4} s_{i}$.

Lemma 4.2 (a) The class $L$ is ample.

(b) The K3 surface $(S, L)$ is Brill-Noether general.

(c) The classes $E_{1}, \ldots, E_{5}$ define elliptic pencils and are the only classes in $\operatorname{Pic}(S)$ of square 0 and degree 4 with respect to $L$.

Proof Let $\Delta=\sum_{i=0}^{4} a_{i} s_{i}$ be an arbitrary class. Then $\Delta^{2}=2 a_{0}-2 \sum_{i=1}^{4} a_{i}$, thus $L . \Delta=$ $8 a_{0}-\Delta^{2}$. If $\Delta$ is effective, then $a_{0}=\frac{1}{2} s_{0} . \Delta \geq 0$ since $s_{0}$ is nef. It follows that $L . \Delta \geq 2$ for any (-2)-curve $\Delta$, and we conclude (a). It also immediately follows that there exists no nontrivial effective class $\Delta$ such that either $\Delta^{2}=0$ and $\Delta . L \leq 3$ or $\Delta^{2}=2$ and $\Delta . L=5$. 
This implies (b) by either a direct computation using the definition of Brill-Noether generality or invoking, e.g., [17, Prop. 10.5] and [32], or [14, Lemma 1.7].

To prove that $\left|E_{i}\right|$ is an elliptic pencil, it suffices to show that $E_{i}$ is nef by [32]. If $E_{i}$ for some $i \in\{1, \ldots, 5\}$ is not nef, there exists a $(-2)$-curve $\Gamma$ with $\Gamma . E_{i} \leq 0$. Let $k:=-\Gamma . E_{i} \geq 1$. Then $\left(E_{i}-k \Gamma\right)^{2}=0$ and $E_{i}-k \Gamma$ is effective and nontrivial with $\left(E_{i}-k \Gamma\right) \cdot L \leq 4-k \leq 3$ by ampleness of $L$, a contradiction to the Brill-Noether generality. Finally, if $F$ is another effective class with $F^{2}=0$, then $F . E_{i} \geq 2$ for all $i$, since $F$ moves in (at least) a pencil. Thus $F \cdot L=\frac{1}{2} F \cdot\left(E_{1}+\cdots+E_{5}\right) \geq 5$.

We will show that the general curve lies on a six-dimensional family of such $K 3$ surfaces of Picard rank 5. We will use the cone over the Grassmannian $G\left(V_{5}, 2\right)$ in $\mathbb{P}^{10}$.

\subsection{K3 sections of a cone of the Grassmannian $G\left(V_{5}, 2\right)$}

Let $\mathfrak{M}$ be the rank 5 lattice above. Let $\mathcal{F}^{\mathfrak{M}}$ be the moduli space of $\mathfrak{M}$-polarized $K 3$ surfaces and $\mathcal{P}^{\mathfrak{M}}$ be as in the introduction. Recall that $\operatorname{dim} \mathcal{F}^{\mathfrak{M}}=15$ and $\operatorname{dim} \mathcal{P}^{\mathfrak{M}}=21$. Also recall that a general genus 6 curve carries precisely five elliptic pencils $\left|A_{1}\right|, \ldots,\left|A_{5}\right|$ of degree four, which satisfy $2 K_{C} \sim A_{1}+\cdots+A_{5}$.

By [5] the moduli space $\mathcal{F}^{\mathfrak{M}}$ is birational to $\mathcal{M}_{6}$, which is well-known to be rational by [34]. More precisely, Artebani and Kondō show that $\mathcal{F}^{\mathfrak{M}}$ is the locus of $K 3$ surfaces admitting a double cover to a quintic Del Pezzo surface branched along a curve of genus 6. In particular, this shows that the moduli map $\psi: \mathcal{P}^{\mathfrak{M}} \rightarrow \mathcal{M}_{6}$ is dominant since we get a section. However, the pairs $(S, L)$ admit automorphisms fixing $L$, therefore $\mathcal{P}^{\mathfrak{M}}$ is not birational to a $\mathbb{P}^{6}$-bundle over $\mathcal{F}^{\mathfrak{M}}$ and one cannot conclude its unirationality from the rationality of $\mathcal{F}^{\mathfrak{M}}$. We will show by our construction that $\mathcal{P}^{\mathfrak{M}}$ is unirational and that $\mathcal{F}^{\mathfrak{M}}$ is the space of polarized $K 3$ surfaces of genus 6 such that all the five $g_{4}^{1}$ s of their smooth curve sections are induced by elliptic pencils on the surfaces.

Theorem 4.3 (a) The moduli map $\psi: \mathcal{P}^{\mathfrak{M}} \rightarrow \mathcal{M}_{6}$ is dominant. Furthermore, a general curve $C$ of genus 6 is a linear section of a smooth $K 3$ surface $S$ such that its five $g_{4}^{1}$ s are induced by five elliptic pencils $\left|E_{1}\right|, \ldots,\left|E_{5}\right|$ on $S$ satisfying $2 C \sim E_{1}+\cdots+E_{5}$.

(b) $\mathcal{P}^{\mathfrak{M}}$ is unirational.

Proof (a) We will describe a $K 3$ surface containing the general curve in $\mathcal{M}_{6}$ as well as the geometry describing the elliptic pencils on the $K 3$ surface. This is based on Mukai's result $[27, \S 6]$.

Let $C \in \mathcal{M}_{6}$ be a general curve of genus 6 which is given as follows. We fix a Plücker embedding of the Grassmannian $G\left(V_{5}, 2\right) \subset \mathbb{P}^{9}$. Then there exists a projective 5 -space $P \subset \mathbb{P}^{9}$ as well as a quadric hypersurface $Q \subset P$ such that $C=P \cap Q \cap G\left(V_{5}, 2\right)$.

Let $P^{\vee}=\mathbb{P}^{3} \subset \mathbb{P}^{9^{\vee}}$ be the dual space. As $C$ is assumed to be general, $W_{4}^{1}(C)$ is finitedimensional, more precisely $W_{4}^{1}(C)$ consists of five smooth points, and is isomorphic to $P^{\vee} \cap G\left(2, V_{5}\right) \subset \mathbb{P}^{9 \vee}$, that is, the intersection of $P^{\vee}$ and the dual Grassmannian $G\left(2, V_{5}\right)=$ $G\left(V_{5}, 2\right)^{\vee} \subset \mathbb{P}^{9^{\vee}}$. By Lemma 4.1 each point of $P^{\vee} \cap G\left(2, V_{5}\right)$ corresponds to a pencil of Grassmannians of type $G(4,2)$ in $\mathbb{P}^{9}$. This pencil induces a cubic scroll in $\mathbb{P}^{9}$ whose restriction to $C$ cuts out the corresponding point of $W_{4}^{1}(C)$.

Now let $\widehat{G\left(V_{5}, 2\right)} \subset \mathbb{P}^{10}$ be the cone over the Grassmannian $G\left(V_{5}, 2\right)$ with vertex point $v$. We denote $\widehat{G\left(2, V_{5}\right)} \subset \mathbb{P}^{10^{\vee}}$ the cone over the dual Grassmannian with vertex $w$ such that $\widehat{G\left(2, V_{5}\right)}=\widehat{G\left(V_{5}, 2\right)}{ }^{\vee}$. We consider the given projective 5-space $P$ as a subspace of $\mathbb{P}^{10}$. 
Let $P_{v}=\overline{P+v}$ be the span of $P$ and the vertex $v$. Let $Q^{\prime} \subset P_{v}$ be a quadric hypersurface such that $Q^{\prime} \cap P=Q$. We get a $K 3$ surface $S=\widehat{G\left(V_{5}, 2\right)} \cap P_{v} \cap Q^{\prime}$, which we can assume to be smooth for general $Q^{\prime}$. Then the dual space of this $P_{v}$ is exactly the above $P^{\vee}$. As above the five intersection points $P^{\vee} \cap \widehat{G\left(V_{5}, 2\right)}=P^{\vee} \cap G\left(V_{5}, 2\right)$ correspond to five pencils of Grassmannians in $\mathbb{P}^{10}$ whose restriction to $S$ are the five elliptic pencils of degree 4 on $S$. We get the desired $K 3$ surface with the right Picard lattice.

(b) Recall that any canonical model of a general curve of genus 6 can be realized as a quadratic section of a fixed quintic Del Pezzo surface $Y \subset \mathbb{P}^{5}$ (see [34]).

We fix a $\mathbb{P}^{6} \supset \mathbb{P}^{5}$ and a point $v \in \mathbb{P}^{6}$. Let $\widehat{Y}$ be the cone over $Y$ with vertex $v$. For a general curve $C \in \mathcal{M}_{6}$ we consider the linear system $\mathfrak{L}_{C}$ of quadratic sections of $\widehat{Y}$ containing $C$. We have $\operatorname{dim} \mathfrak{L}_{C}=h^{0}\left(\mathbb{P}^{6}, \mathcal{O}_{\mathbb{P}^{6}}(2)\right)-h^{0}\left(\mathbb{P}^{5}, \mathcal{O}_{\mathbb{P}^{5}}(2)\right)-1=6$. We define the incidence correspondence

$$
I=\{(C, S) \mid C \subset S\} \subset\left|\mathcal{O}_{Y}(2)\right| \times\left|\mathcal{O}_{\widehat{Y}}(2)\right|=\mathbb{P}^{15} \times \mathbb{P}^{22}
$$

together with the projection $\pi: I \rightarrow\left|\mathcal{O}_{Y}(2)\right|$, whose fibers are given by $\mathfrak{L}_{C}$. It follows that $\pi$ has the structure of a $\mathbb{P}^{6}$-bundle, whence $\operatorname{dim}(I)=15+6=21$.

By the proof of part (a) the general member of $\mathfrak{L}_{C}$ is a smooth $K 3$ surface in $\mathcal{F}^{\mathfrak{M}}$ (note that $P=\mathbb{P}^{5}, P_{v}=\mathbb{P}^{6}, Y=P \cap G\left(V_{5}, 2\right)$ and $\widehat{Y}=\widehat{G\left(V_{5}, 2\right)} \cap P_{v}$ in the notation of that proof). Hence, we get a natural rational moduli map $\varphi: I \rightarrow \mathcal{P}^{\mathfrak{M}}$. Since $I$ is unirational, the corollary will follow if we prove that $\varphi$ is dominant, equivalently, generically finite, since $\mathcal{P}^{\mathfrak{M}}$ is irreducible of the same dimension as $I$.

Assume therefore that $\varphi$ has positive-dimensional fibers. Since the rational moduli map $\left|\mathcal{O}_{Y}(2)\right| \rightarrow \mathcal{M}_{6}$ is finite, the fibers of $\varphi$ lie in fibers of $\pi$. Hence, the $K 3$ surfaces in $\mathfrak{L}_{C}$ do not have maximal variation in moduli. Note that $\mathfrak{L}_{C}$ contains the quadratic sections of the form $Y \cup Y^{\prime}$ where $Y^{\prime} \in \mathbb{P} H^{0}\left(\widehat{Y}, \mathcal{O}_{\widehat{Y}}(1)\right)$ which form a hypersurface in $\mathfrak{L}_{C}$. Hence a general one-dimensional family in $\mathfrak{L}_{C}$ is non-isotrivial, a contradiction.

Remark 4.4 (a) The proof of Corollary 4.3 shows that our construction dominates the moduli space $\mathcal{F}^{\mathfrak{M}}$, that is, the general $K 3$ surface in $\mathcal{F}^{\mathfrak{M}}$ is a quadratic section of a cone over a quintic Del Pezzo surface in $\mathbb{P}^{5}$.

(b) By [27], all Brill-Noether general $K 3$ surfaces of genus 6 can be realized as a quadratic section of either a smooth quintic Del Pezzo threefold in $\mathbb{P}^{6}$ or a cone over a quintic Del Pezzo surface. Item (a) shows that $\mathcal{F}^{\mathfrak{M}}$ is precisely the locus of $K 3$ surfaces that cannot be realized in a smooth Del Pezzo threefold.

\section{Lazarsfeld-Mukai bundles and their stability}

For $K 3$ surfaces constructed in Sects. 3 and 4 we will show that these are $K 3$ surfaces without any stable rank 2 Lazarsfeld-Mukai bundle with determinant $L$ and $c_{2}=3$ or 4, respectively. This shows in particular that the result of Lelli-Chiesa [23, Thm. 4.3] about stability of rank 2 vector bundles on $K 3$ surfaces is optimal.

We recall the definition and basic properties of Lazarsfeld-Mukai bundles, which will also be needed in Sect. 6. Let $S$ be a $K 3$ surface and let $C \subset S$ be a smooth curve of genus $g$ with a globally generated line bundle $A$ of degree $d$ with $h^{0}(C, A)=r+1$. The Lazarsfeld-Mukai bundle $\mathcal{E}_{C, A}$ is defined via an elementary transformation on $S$ :

$$
0 \longrightarrow \mathcal{E}_{C, A}^{\vee} \longrightarrow H^{0}(C, A) \otimes \mathcal{O}_{S} \longrightarrow A \longrightarrow 0
$$


where $A$ is considered as a coherent sheaf on $S$ supported on $C$. Hence, it is a bundle of rank $r+1$ satisfying $c_{1}\left(\mathcal{E}_{C, A}\right)=[C], c_{2}\left(\mathcal{E}_{C, A}\right)=\operatorname{deg} A=d$ and $H^{i}\left(S, \mathcal{E}_{C, A}\right)=0$ for $i=1,2$. The bundles have been introduced by Lazarsfeld [22] and Mukai [30]. Dualizing the above sequence, we get

$$
0 \longrightarrow H^{0}(C, A)^{*} \otimes \mathcal{O}_{S} \longrightarrow \mathcal{E}_{C, A} \longrightarrow \omega_{C} \otimes A^{*} \longrightarrow 0
$$

and in particular a distinguished $(r+1)$-dimensional subspace $H^{0}(C, A)^{*} \subset H^{0}\left(\mathcal{E}_{C, A}\right)$. Equivalently, by [2, Prop. 1.3], a rank $(r+1)$-bundle $\mathcal{E}$ on $S$ is a Lazarsfeld-Mukai bundle if and only if $h^{1}(S, \mathcal{E})=h^{2}(S, \mathcal{E})=0$ and there exists an $(r+1)$-dimensional subspace $V \subset H^{0}(S, \mathcal{E})$ such that the degeneracy locus of the evaluation morphism $V \otimes \mathcal{O}_{S} \rightarrow \mathcal{E}$ is a smooth curve.

Lemma 5.1 If $A \in W_{d}^{1}(C)$ with $d \leq g-1$ is induced by an elliptic pencil $|E|$ on the $K 3$ surface $S$, then $\mathcal{E}_{C, A}$ is not L-stable, where $L=\mathcal{O}_{S}(C)$. Furthermore, the bundle $\mathcal{E}_{C, A}$ is L-unstable, if $d<g-1$.

Proof This is essentially already contained in [1, Proof of Thm. 1.1]. Using the snake lemma, we get the following commutative diagram

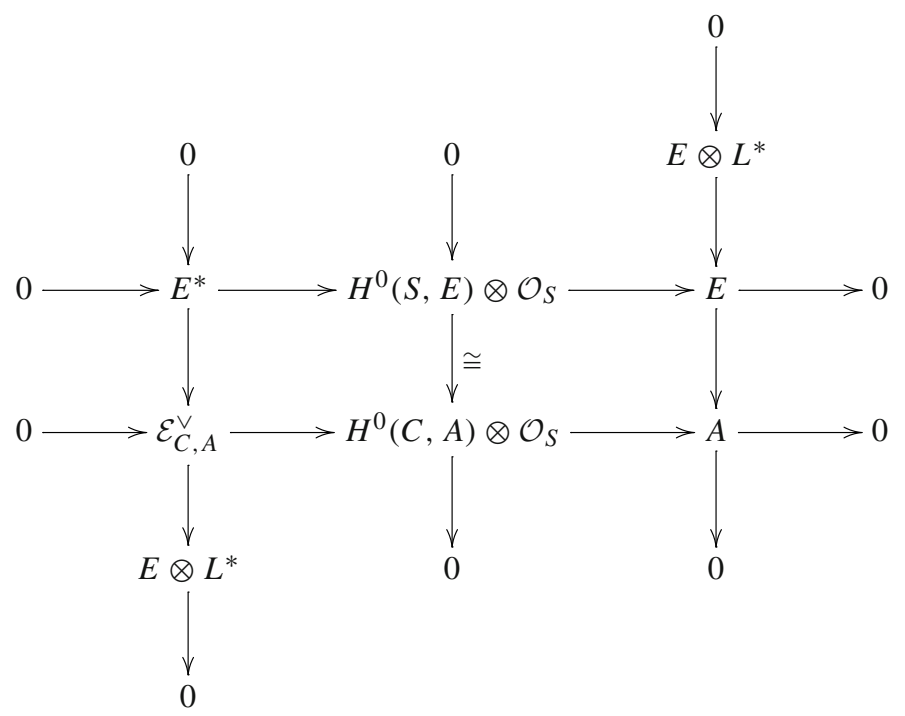

Dualizing the left column, we see that $L \otimes E^{*}$ is a subbundle of $\mathcal{E}_{C, A}$. Computing slopes, we get $\mu\left(L \otimes E^{*}\right)=2 g-2-d \geq g-1=\mu\left(\mathcal{E}_{C, A}\right)$.

Corollary 5.2 Let $(S, L) \in \mathcal{F}_{4}^{\mathfrak{U}(3)}$ be a Brill-Noether general polarized K3 surface as in Sect. 3.1. Then $S$ contains only L-strictly semistable Lazarsfeld-Mukai bundles $\mathcal{E}_{C, A}$ of rank 2 and $\operatorname{det}\left(\mathcal{E}_{C, A}\right)=L, c_{2}\left(\mathcal{E}_{C, A}\right)=3$ for $C \in|L|$ smooth.

Proof Note that $W_{3}^{1}(C)$ consists of exactly two residual pencils of divisors which extend to two elliptic pencils on $S$. We can apply Lemma 5.1, and the corollary follows.

Corollary 5.3 Let $(S, L) \in \mathcal{F}_{6}^{\mathfrak{M}}$ be a Brill-Noether general polarized $K 3$ surface as in Sect. 4. Then $S$ contains only L-unstable Lazarsfeld-Mukai bundles $\mathcal{E}_{C, A}$ of rank 2 and $\operatorname{det}\left(\mathcal{E}_{C, A}\right)=L, c_{2}\left(\mathcal{E}_{C, A}\right)=4$ for $C \in|L|$ smooth. 
Proof Since $C$ is Brill-Noether general, every pencil in $W_{4}^{1}(C)$ is induced by an elliptic pencil on the $K 3$ surface $S$. The result follows from Lemma 5.1 .

Remark 5.4 Part (i) of [23, Thm. 4.3] implies that on any Brill-Noether general $K 3$ surface $(S, L)$ of genus $g$ there are $L$-stable Lazarsfeld-Mukai bundles of determinant $L$ and $c_{2}$ equal to $d$ as soon as $\rho(g, 1, d)>0$. (Indeed, sections of Brill-Noether general $K 3$ surfaces have maximal gonality as a consequence of the definition and have Clifford dimension 1 by ampleness of $L$, cf. [18, Thm. 1.2] or [10, Prop. 3.3]). The above corollaries show that this does not always hold for $\rho(g, 1, d)=0$ (at least when $g=4$ or 6 ).

\section{K3 surfaces of genus 8}

In this section we construct $K 3$ surfaces of genus 8 with the maximal number of elliptic pencils of degree 5. We recall Mukai's construction from [27,29] and fix our notation.

Let $(S, L)$ be a Brill-Noether general polarized $K 3$ surface of genus 8 . Then there exists a unique globally generated stable vector bundle $\mathcal{E}$ of rank 2 with determinant $L$ and Euler characteristic 6 (this can be constructed as the Lazarsfeld-Mukai bundle associated to a $g_{5}^{1}$ on any smooth $C \in|L|$ not induced by an elliptic pencil on $S$ by [2, Prop. 1.3]). It is known that $V_{6}=H^{0}(S, \mathcal{E})$ is six-dimensional. Every fiber $\mathcal{E}_{s}$ of $\mathcal{E}$ for $s \in S$ is a 2-dimensional quotient space of $V_{6}$, which induces a morphism $\phi_{\mathcal{E}}: S \rightarrow G\left(V_{6}, 2\right), s \mapsto \mathcal{E}_{s}$. The Grassmannian $G\left(V_{6}, 2\right)$ is naturally embedded into $\mathbb{P}^{*}\left(\bigwedge^{2} V_{6}\right)=\mathbb{P}^{14}$ via the Plücker embedding. The second exterior product induces a surjective map on global sections

$$
\lambda: \bigwedge^{2} H^{0}(S, \mathcal{E}) \rightarrow H^{0}\left(S, \bigwedge^{2} \mathcal{E}\right)
$$

and we get the following commutative diagram

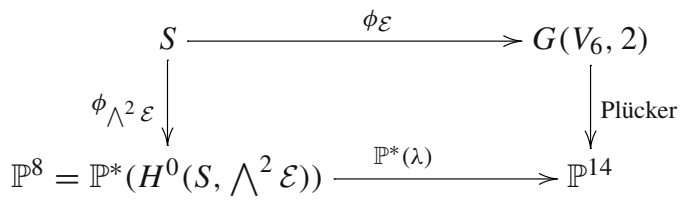

where $\mathbb{P}^{*}(\lambda)$ is the linear embedding induced by $\lambda$. Since $\bigwedge^{2} \mathcal{E}=c_{1}(\mathcal{E})=L$, the map $\phi_{\bigwedge^{2}} \mathcal{E}$ is given by the linear system $|L|$. The above diagram is cartesian, that is, $S=\mathbb{P}^{8} \cap G\left(V_{6}, 2\right)$.

Hyperplane sections of $G\left(V_{6}, 2\right)$ are parametrized by $\mathbb{P}_{*}\left(\bigwedge^{2} V_{6}\right)$. The dual of $\mathbb{P}^{8}$ is a five-dimensional projective space $\mathbb{P}^{5}=\mathbb{P}_{*}(\operatorname{ker} \lambda) \subset \mathbb{P}_{*}\left(\bigwedge^{2} V_{6}\right)$.

Let $C \in|L|$ be a smooth curve. The Brill-Noether generality of $(S, L)$ is equivalent to $C$ not containing a $g_{7}^{2}$ (arguing as in [13,22] or see [14, Lemma 1.7]). Let $\mathcal{E}_{C}$ be the restriction of $\mathcal{E}$ to $C$, which is stable by [27, §3] and $H^{0}(S, \mathcal{E}) \cong H^{0}\left(C, \mathcal{E}_{C}\right)$. As above we get a surjective morphism $\lambda_{C}: \bigwedge^{2} H^{0}\left(C, \mathcal{E}_{C}\right) \rightarrow H^{0}\left(C, \omega_{C}\right)$ and a commutative cartesian diagram

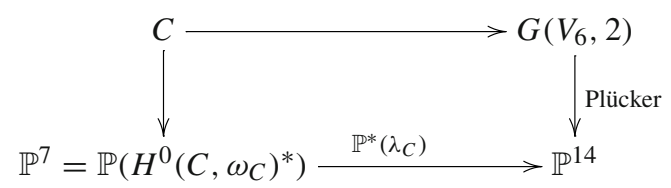

since $\mathbb{P}_{*}\left(\lambda_{C}\right) \cap G\left(2, V_{6}\right) \cong W_{5}^{1}(C)$ is finite (see [27, Thm. C]). Note that $\mathbb{P}_{*}\left(\lambda_{C}\right)$ is a six-dimensional space containing $\mathbb{P}_{*}(\lambda)$. 
For our purpose we state Mukai's result in the following form.

Lemma 6.1 (Mukai) A linear intersection of $G\left(V_{6}, 2\right)$ and $\mathbb{P}^{8}$ is a surface (in particular a Brill-Noether general $K 3$ surface if smooth) if and only if the dual projective space $\mathbb{P}^{5}$ intersects the Grassmannian $G\left(2, V_{6}\right)$ in the following way: for every $\mathbb{P}^{6} \supset \mathbb{P}^{5}$ the intersection with $G\left(2, V_{6}\right) \subset \mathbb{P}_{*}\left(\bigwedge^{2} V_{6}\right)$ is finite.

Proof The "only if" part follows from the above. Conversely, the second condition is equivalent to any hyperplane section of the given linear section being a curve.

\subsection{Linear sections of $G\left(V_{6}, 2\right)$ and elliptic pencils}

We are interested in $K 3$ surfaces $S \subset \mathbb{P}^{8}$ with an elliptic pencil of minimal degree 5 . We describe a way of constructing such $K 3$ surfaces.

We use the notation above. Let $V_{6}$ be a 6-dimensional complex vector space, and let $V_{5}$ be a 5-dimensional subspace of $V_{6}$. We consider $G\left(V_{5}, 2\right) \subset G\left(V_{6}, 2\right) \subset \mathbb{P}^{*}\left(\bigwedge^{2} V_{6}\right)$. By a dimension count, a general 8-dimensional linear subspace of $\mathbb{P}^{14}$ intersects $G\left(V_{5}, 2\right)$ in 5 points. Assume instead that our $\mathbb{P}^{8}$ intersects $G\left(V_{6}, 2\right)$ transversally and $\mathbb{P}^{8} \cap G\left(V_{5}, 2\right)$ is a smooth curve, which is then an irreducible elliptic normal curve of degree 5 . Then we get a $K 3$ surface $S$ with an elliptic pencil.

\subsubsection{Dual Grassmannian and Schubert varieties}

Even more is true. As Mukai already notices in [27, end of p.3], a hyperplane corresponds to a point in the dual Grassmannian $G\left(2, V_{6}\right) \subset \mathbb{P}_{*}\left(\bigwedge^{2} V_{6}\right)$ if and only if it cuts out a Schubert subvariety. We will explain this fact in detail.

Let $U \in G\left(2, V_{6}\right)$ be a point in the Grassmannian, that is, $U \subset V_{6}$ be a 2-dimensional subspace of $V_{6}$. Hence, $U^{\perp}=V_{6} / U$ is a 4-dimensional quotient of $V_{6}$. By the perfect pairing $\bigwedge^{2} V_{6} \otimes \wedge^{4} V_{6} \rightarrow \mathbb{C}$ we may interpret $U^{\perp}$ as a linear function on $\bigwedge^{2} V_{6}$, denoted by $H_{U}$. We compute the hyperplane section $H_{U} \cap G\left(V_{6}, 2\right)$. By definition $H_{U}: \operatorname{ker}\left(\bigwedge^{2} V_{6} \stackrel{\wedge^{4} U^{\perp}}{\longrightarrow}\right.$ $\left.\wedge^{6} V_{6}=\mathbb{C}\right)$. Thus,

$$
\begin{aligned}
H_{U} \cap G\left(V_{6}, 2\right) & =\left\{U^{\prime} \in G\left(V_{6}, 2\right) \mid \bigwedge^{2} U^{\prime} \wedge \bigwedge^{4} U^{\perp}=0\right\} \\
& =\left\{U^{\prime} \in G\left(V_{6}, 2\right) \mid \operatorname{dim}\left(U^{\prime} \cap U^{\perp}\right) \geq 1\right\}=: \Sigma_{1}\left(U^{\perp}\right)
\end{aligned}
$$

is a Schubert variety. Note that $\operatorname{dim}\left(U^{\prime} \cup U^{\perp}\right) \leq 5$ for $U^{\prime} \in H_{U} \cap G\left(V_{6}, 2\right)$, and it is easy to check that

$$
\Sigma_{1}\left(U^{\perp}\right)=\bigcup_{v \in W} G\left(U^{\perp} \cup v, 2\right),
$$

where $W \oplus U^{\perp}=V_{6}$. Note that everything is compatible with projectivization. Finally, we see that $\mathbb{P}^{*}\left(H_{U}\right) \cap G\left(V_{6}, 2\right) \subset \mathbb{P}^{14}$ is a pencil of Grassmannians of type $G(5,2)$. The converse direction can be shown similarly.

We conclude that every intersection point of $\mathbb{P}_{*}(\operatorname{ker} \lambda) \cap G\left(2, V_{6}\right)$ gives a pencil of elliptic curves on $S$. In order to get $K 3$ surfaces with many elliptic pencils of degree 5, we have to construct a transversal linear section $\mathbb{P}^{8}$ such that its dual $\mathbb{P}_{*}(\operatorname{ker} \lambda)$ intersects the Grassmannian $G\left(2, V_{6}\right)$ in as many points as possible. 


\subsubsection{Extension of elliptic curves to the Grassmannian $G\left(V_{6}, 2\right)$}

Let $(S, L)$ be a Brill-Noether general polarized $K 3$ surface of genus 8 with an elliptic pencil $|E|$ satisfying $L . E=5$. As $S$ can be embedded (as a linear section) into the Grassmannian $G\left(V_{6}, 2\right)$, we will show that every elliptic curve $E^{\prime} \in|E|$ is a linear section of a subGrassmannian of type $G(5,2)$ of $G\left(V_{6}, 2\right)$.

We need some lemmas. We note that $(L-E)^{2}=4$ and $(L-E) \cdot L=9$, whence $h^{0}(L-E) \geq 4$ by Serre duality and Riemann-Roch.

Lemma 6.2 The complete linear system $|L-E|$ is base point free and maps $S$ birationally onto a quartic surface in $\mathbb{P}^{3}$ having at most isolated $A_{1}$-singularities coming from contractions of smooth rational curves $\Gamma$ satisfying $\Gamma . L=\Gamma . E=1$.

Proof Assume there exists an effective divisor $\Delta$ such that $\Delta^{2}=-2$ and $\Delta .(L-E) \leq 0$. In particular, $\Delta . E \geq \Delta . L>0$. Then $(L-E-\Delta)^{2} \geq 2$, whence $h^{0}(L-E-\Delta) \geq 3$. As $(S, L)$ is assumed to be Brill-Noether general, we must have $h^{0}(E+\Delta)=h^{0}(E)=2$, thus $\Delta . E=1$, and consequently $\Delta . L=1$ and $\Delta .(L-E)=0$. It follows that $L-E$ is nef. It also follows, once we have proved that $|L-E|$ defines a birational morphism, that any connected curve contracted by this morphism is an irreducible rational curve of degree one with respect to $L$ and $E$, proving that the image surface has at most isolated rational $A_{1}$-singularities.

To prove that $|L-E|$ defines a birational morphism, it suffices by the well-known results of Saint-Donat [32] to prove that there is no irreducible curve $D$ on $S$ satisfying $D^{2}=0$ and $D$. $(L-E)=1$ or 2 . If such a $D$ exists, then it is easily seen to satisfy $D . L \geq 5$ by BrillNoether generality. Hence, D. $E \geq 3$, so that $(D+E)^{2} \geq 6$. It follows that $h^{0}(D+E) \geq 5$. Since $(L-E-D)^{2} \geq 0$ and $(L-E-D) . D \geq 1$, we have $h^{0}(L-E-D) \geq 2$ by Riemann-Roch and Serre duality, contradicting Brill-Noether generality.

Let $C \in|L|$ be a smooth curve and let $\mathcal{E}=\mathcal{E}_{C, A}$ be the Lazarsfeld-Mukai bundle associated to $C$ and a pencil $|A|$ of degree 5 on $C$. Note that the bundle $\mathcal{E}_{C, A}$ is the unique $L$-stable bundle on $S$ with determinant $L$ and Euler characteristic 6. We write $A_{E}=E \otimes \mathcal{O}_{C}$ and note that $A \nsubseteq A_{E}$ by Lemma 5.1 .

Lemma 6.3 Let $(S, L), E$ and $\mathcal{E}=\mathcal{E}_{C, A}$ be as above. Then $h^{0}(\mathcal{E}(-E))=1$ and $h^{1}(\mathcal{E}(-E))=h^{2}(\mathcal{E}(-E))=0$. In particular, $H^{0}\left(\left.\mathcal{E}\right|_{E}\right)$ is a five-dimensional quotient of $H^{0}(S, \mathcal{E})$.

Proof Since we know that $h^{0}(\mathcal{E})=6$, the last assertion immediately follows from the claimed cohomology of $\mathcal{E}(-E)$ by the obvious restriction sequence.

We will compute the cohomology of $\mathcal{E}(-E)$ using Serre duality and the sequence

$$
0 \longrightarrow \mathcal{E}^{\vee}(E) \longrightarrow H^{0}(C, A) \otimes \mathcal{O}_{S}(E) \longrightarrow A \otimes A_{E} \longrightarrow 0,
$$

which is (5.1) tensored by $\mathcal{O}_{S}(E)$.

Since $\mathcal{E}^{\vee}(E)$ is semi-stable of degree -4 , one has $h^{0}\left(\mathcal{E}^{\vee}(E)\right)=0$. Moreover, $h^{0}\left(\mathcal{O}_{S}(E)\right)=2$ and $h^{1}\left(\mathcal{O}_{S}(E)\right)=h^{2}\left(\mathcal{O}_{S}(E)\right)=0$, as $E$ is an irreducible elliptic curve. Hence, the desired cohomology of $\mathcal{E}(-E)$ will follow once we prove that

$$
h^{0}\left(C, A \otimes A_{E}\right)=4 \text { and } h^{1}\left(C, A \otimes A_{E}\right)=1 .
$$

To prove the latter, note that $h^{0}\left(C, A \otimes A_{E}\right)=\chi\left(C, A \otimes A_{E}\right)+h^{1}\left(C, A \otimes A_{E}\right)=3+$ $h^{1}\left(C, A \otimes A_{E}\right)$ by Riemann-Roch. Since $A \supsetneqq A_{E}$, we have $h^{0}\left(C, A \otimes A_{E}\right) \geq 4$; moreover, 
equality must hold, as otherwise $h^{0}\left(C, \omega_{C} \otimes\left(A \otimes A_{E}\right)^{-1}\right)=h^{1}\left(C, A \otimes A_{E}\right) \geq 2$ and $\operatorname{deg}\left(\omega_{C} \otimes\left(A \otimes A_{E}\right)^{-1}\right)=4$, hence $C$ would contain a $g_{4}^{1}$, a contradiction to Brill-Noether generality. This proves (6.2).

By abuse of notation, let $E$ be an elliptic curve of the pencil $|E|$ on $S$. Since $H^{0}\left(\left.\mathcal{E}\right|_{E}\right)$ is a 5-dimensional quotient space of $V_{6}=H^{0}(S, \mathcal{E})$, each fiber $\mathcal{E}_{S}$ for $s \in E$ is a 2-dimensional quotient of $H^{0}\left(\left.\mathcal{E}\right|_{E}\right)$ and hence of $V_{6}$. The image $\phi_{\mathcal{E}}(E)$ of the elliptic curve is contained in $G\left(H^{0}\left(\left.\mathcal{E}\right|_{E}\right), 2\right)$. Since $\lambda$ is surjective and $E$ is projectively normal, we have the following commutative diagram

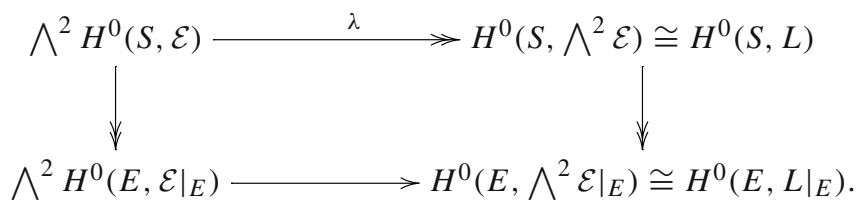

So, we obtain the commutative diagram

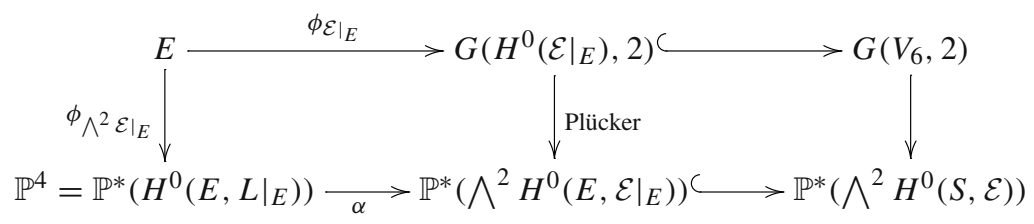

where $\alpha$ is an embedding. The diagram is also cartesian. Indeed, let $\mathbb{P}^{4}=\bar{E}$ be the linear span, then

$$
E \subset \mathbb{P}^{4} \cap G\left(H^{0}\left(\left.\mathcal{E}\right|_{E}\right), 2\right) \subset \mathbb{P}^{4} \cap G\left(V_{6}, 2\right)=\mathbb{P}^{4} \cap \mathbb{P}^{8} \cap G\left(V_{6}, 2\right)=S \cap \mathbb{P}^{4} .
$$

But $E=S \cap \mathbb{P}^{4}$ since $|E|$ and $|L-E|$ are base point free (c.f. Lemma 6.2). Hence, it follows that $E=\mathbb{P}^{4} \cap G\left(H^{0}\left(\left.\mathcal{E}\right|_{E}\right), 2\right)$. By Section 6.1.1, the elliptic pencil $|E|$ on $S$ is cut out by the Schubert cycle $\Sigma_{1}\left(V_{4}\right)$ on $G\left(V_{6}, 2\right)$ for some four-dimensional quotient $V_{4}$. Recall further that there is a one-to-one correspondence between such Schubert cycles and points on the dual Grassmannian $G\left(2, V_{6}\right)$.

The following corollary follows immediately from our discussion.

Corollary 6.4 Let $(S, L)$ be a Brill-Noether general polarized $K 3$ surface of genus 8 . Let $\mathbb{P}_{(S)}^{5} \subset \mathbb{P}_{*}\left(\bigwedge^{2} H^{0}(S, \mathcal{E})\right)$ be the dual space of $\mathbb{P}^{8}=\mathbb{P}^{*} H^{0}(S, L) \subset \mathbb{P}^{*}\left(\bigwedge^{2} H^{0}(S, \mathcal{E})\right)$. There is a one-to-one correspondence between elliptic pencils $|E|$ on S satisfying L.E $=5$ and points of $G\left(2, V_{6}\right) \cap \mathbb{P}_{(S)}^{5}$.

\subsubsection{Maximal number of distinct elliptic pencils}

Let $(S, L)$ be a Brill-Noether general $K 3$ surface of genus 8, and let $E_{1}, E_{2}$ be two classes with $E_{1}^{2}=E_{2}^{2}=0$ and $E_{1} \cdot L=E_{2} \cdot L=5$. Then $E_{1} \cdot E_{2}=2$. Indeed, the Hodge Index Theorem on $E_{1}+E_{2}$ and $L$ yields $E_{1} \cdot E_{2} \leq 3$. Equality implies $\left(E_{1}+E_{2}\right)^{2}=6$ and $\left(L-E_{1}-E_{2}\right)^{2}=0$, whence $h^{0}\left(S, E_{1}+E_{2}\right) \geq 5$ and $h^{0}\left(S, L-E_{1}-E_{2}\right) \geq 2$, a contradiction to Brill-Noether generality.

On can also see this fact geometrically using the notation of the previous section. Let $V_{5}, V_{5}^{\prime}$ be two distinct 5-dimensional quotients of $V_{6}$. The intersection of the Grassmannians $G\left(V_{5}, 2\right)$ and $G\left(V_{5}^{\prime}, 2\right)$ is the Grassmannian $G\left(V_{5} \cap V_{5}^{\prime}, 2\right)$. The Grassmannian $G\left(V_{5} \cap V_{5}^{\prime}, 2\right)$ 
is a 4-dimensional quadric. Hence, if $\mathbb{P}^{8}$ is a general linear subspace such that its intersection with $G\left(V_{5}, 2\right)$ and $G\left(V_{5}^{\prime}, 2\right)$ are elliptic curves, then these elliptic curves intersect in two points, namely $\mathbb{P}^{8} \cap G\left(V_{5} \cap V_{5}^{\prime}, 2\right)$.

If all our above assumptions are satisfied, we get a $K 3$ surface with Picard lattice containing the following lattice

$$
\left(\begin{array}{ccccc}
14 & 5 & 5 & \ldots & 5 \\
5 & 0 & 2 & \ldots & 2 \\
5 & 2 & 0 & \ddots & \vdots \\
\vdots & \vdots & \ddots & \ddots & 2 \\
5 & 2 & \ldots & 2 & 0
\end{array}\right)
$$

An easy computation shows that the maximal possible rank is 10 (otherwise the matrix has at least two positive eigenvalues). Let $\mathfrak{G}_{9}$ be such a lattice of maximal possible rank which is given by the following intersection matrix

$$
\mathfrak{G}_{9}=\underbrace{\left(\begin{array}{ccccc}
14 & 5 & 5 & \ldots & 5 \\
5 & 0 & 2 & \ldots & 2 \\
5 & 2 & 0 & \ddots & \vdots \\
\vdots & \vdots & \ddots & \ddots & 2 \\
5 & 2 & \ldots & 2 & 0
\end{array}\right)}_{10 \text { columns }} .
$$

We denote $S$ a $K 3$ surface with the above Picard lattice $\mathfrak{G}_{9}$ of rank 10 (which again exists by [24, Thm. 2.9(i)] or [31]), and let $L$ be the basis element of square 14, which can be taken to be big and nef by standard arguments (see [7, VIII, Prop. 3.10]). Let $E_{i}, i=1, \ldots, 9$, be the generators of square zero.

Lemma 6.5 (a) The class L is ample.

(b) The K3 surface $(S, L)$ is Brill-Noether general.

(c) The classes $E_{1}, \ldots, E_{9}$ define elliptic pencils.

This can probably be proved arguing as in the proof of Lemma 4.2, but the computations are much more tedious. Instead we will give a constructive proof in the next subsection.

\subsection{A unirational construction of $K 3$ surfaces with nine distinct elliptic pencils}

Recall that any projective equivalence of two $K 3$ surfaces that are linear sections of the Grassmannian $G\left(V_{6}, 2\right)$ is induced by an automorphism of $V_{6}$ (see [28, Theorem 0.2]).

By Corollary 6.4, any Brill-Noether general polarized $K 3$ surface $S$ of genus 8 with exactly nine elliptic pencils of degree five induces and is induced by a unique five-dimensional space $\mathbb{P}_{(S)}^{5}$ intersecting $G\left(2, V_{6}\right) \subset \mathbb{P}^{14}$ in exactly nine points. We reformulate this fact in the following proposition. To state it we denote $\mathcal{H}_{9,5}\left(G\left(2, V_{6}\right)\right)$ the space of 9-secant 5planes of the Grassmannian $G\left(2, V_{6}\right) \subset \mathbb{P}^{14}$ intersecting the latter in exactly nine points and $\widetilde{\mathcal{H}}_{9,5}\left(G\left(2, V_{6}\right)\right)$ this space modulo the automorphisms of $V_{6}$.

Proposition 6.6 The moduli space of Brill-Noether general polarized K3 surfaces of genus 8 with exactly nine elliptic pencils of degree 5 is birational to $\widetilde{\mathcal{H}}_{9,5}\left(G\left(2, V_{6}\right)\right)$, and both spaces are non-empty. 
Proof By Corollary 6.4, we only need to prove the non-emptiness of $\mathcal{H}_{9,5}\left(G\left(2, V_{6}\right)\right)$. A general intersection of $G\left(2, V_{6}\right)$ and a $\mathbb{P}^{7}$ is a smooth curve $C$ of genus 8 and the general curve of genus 8 is obtained in this way (cf. [27]). Furthermore, a 9-secant 5-plane of $G\left(2, V_{6}\right)$ contained in this $\mathbb{P}^{7}$ is also a 9 -secant of $C$, which is a divisor in a $g_{9}^{3}$ by the geometric Riemann-Roch. Note that the $g_{9}^{3}$ is automatically base point free as otherwise the curve would not be Brill-Noether general and thus could not be a linear section of the $G\left(2, V_{6}\right)$ by [27]. Hence a general divisor in the $g_{9}^{3}$ induces an element of $\mathcal{H}_{9,5}\left(G\left(2, V_{6}\right)\right)$.

We have reduced the problem to constructing a curve of genus 8 as a linear section of $G\left(2, V_{6}\right)$ carrying a $g_{9}^{3}$, or equivalently, taking residuals, a $g_{5}^{1}$. Such a curve can be realized as follows: We get a divisor $D$ of degree 5 in a $g_{5}^{1}$ on a curve $C$ of genus 8 if we fix a $G\left(2, V_{5}\right)$ (where $V_{5}$ is a 5-dimensional subspace of $V_{6}$ ) and choose a $\mathbb{P}^{7}$ such that $C=\mathbb{P}^{7} \cap G\left(2, V_{6}\right)$ and $D=\mathbb{P}^{7} \cap G\left(2, V_{5}\right)$ induces the $g_{5}^{1}=|D|$. In an ancillary file, cf. [15], we have implemented this construction in Macaulay2 (see [12]) as well as the construction of the corresponding K3 surface.

The Picard lattice of the $K 3$ surfaces in the moduli space in Proposition 6.6 contains the lattice $\mathfrak{G}_{9}$ and the generator of square 14 is (very) ample and the generators of square 0 are nef. Let $\mathcal{F}^{\mathfrak{G} 9}$ be the moduli space of $\mathfrak{G}_{9}$-lattice polarized $K 3$ surfaces. By standard deformation arguments (see [20, Thm. 14]) the very general element in $\mathcal{F}^{\mathfrak{G} 9}$ has Picard lattice equal to $\mathfrak{G}_{9}$, is Brill-Noether general with ample generator of square 14 and the generators of square 0 define elliptic pencils.

Proof of Lemma 6.5 The last discussion proves the lemma for the very general element in $\mathcal{F}^{\mathfrak{G}_{9}}$ having Picard lattice equal to $\mathfrak{G}_{9}$. Since the properties (a)-(c) of the lemma only depend on the lattice, this finishes the proof.

We also have the following

Theorem 6.7 The moduli space $\mathcal{F}^{\mathfrak{G}_{9}}$ of $\mathfrak{G}_{9}$-lattice polarized $K 3$ surfaces is unirational.

Proof The above discussion shows that $\mathcal{F}^{\mathfrak{G}_{9}}$ is birational to $\tilde{\mathcal{H}}_{9,5}\left(G\left(2, V_{6}\right)\right)$. In particular, $\widetilde{\mathcal{H}}_{9,5}\left(G\left(2, V_{6}\right)\right)$ is irreducible.

Consider the following incidence variety

$$
\begin{aligned}
& \left\{\left(V_{5}^{9}, \mathbb{P}^{7}\right) \in \mathcal{H}_{9,5}\left(G\left(2, V_{6}\right)\right) \times G\left(8, \Lambda^{2} V_{6}\right) \mid V_{5}^{9} \subset \mathbb{P}^{7},\right. \\
& \left.C=\mathbb{P}^{7} \cap G\left(2, V_{6}\right) \text { a smooth curve }\right\}
\end{aligned}
$$

and denote $I$ its quotient with the automorphisms of $V_{6}$ acting diagonally. Then $I$ admits a natural first projection map $\pi_{1}: I \rightarrow \widetilde{\mathcal{H}}_{9,5}\left(G\left(2, V_{6}\right)\right)$ and a second projection to the moduli space of curves of genus 8 . As for $K 3$ surfaces, any projective equivalence of two curves of genus 8 that are linear sections of the Grassmannian $G\left(2, V_{6}\right)$ is induced by an automorphism of $V_{6}$.

The proof of Proposition 6.6 shows that $I$ is non-empty and is therefore birational to a $\mathbb{P}^{3}$-bundle over the universal Brill-Noether variety $\mathcal{W}_{8,9}^{3}$ by the universal Abel-Jacobi map. Hence, $I$ is unirational and irreducible since $\mathcal{W}_{8,9}^{3} \cong \mathcal{W}_{8,5}^{1}$ is unirational (and irreducible) by [3]. Since $\pi_{1}$ is dominant (because $\widetilde{\mathcal{H}}_{9,5}\left(G\left(2, V_{6}\right)\right)$ is irreducible), $\widetilde{\mathcal{H}}_{9,5}\left(G\left(2, V_{6}\right)\right)$ is unirational. The theorem follows. 
One may also consider, for $i \in\{0, \ldots, 8\}$, the moduli spaces $\mathcal{F}^{\mathfrak{G}_{i}}$ of $\mathfrak{G}_{i}$-lattice polarized $K 3$ surfaces, where $\mathfrak{G}_{i}$ is the rank $i+1$ lattice

$$
\mathfrak{G}_{i}=\underbrace{\left(\begin{array}{ccccc}
14 & 5 & 5 & \ldots & 5 \\
5 & 0 & 2 & \ldots & 2 \\
5 & 2 & 0 & \ddots & \vdots \\
\vdots & \vdots & \ddots & \ddots & 2 \\
5 & 2 & \ldots & 2 & 0
\end{array}\right)}_{i+1 \text { columns }}
$$

Then $\operatorname{dim} \mathcal{F}^{\mathfrak{G}_{i}}=19-i$ and $\mathcal{F}^{\mathfrak{G}_{i+1}} \subset \mathcal{F}^{\mathfrak{G}_{i}}$ for each $i \in\{0, \ldots, 8\}$. Note that $\mathcal{F}^{\mathfrak{G}_{0}}=\mathcal{F}_{8}$

Theorem 6.8 The moduli spaces $\mathcal{F}^{\mathfrak{G}_{i}}$ of $\mathfrak{G}_{i}$-lattice polarized $K 3$ surfaces are unirational for $i \leq 6$.

Proof The case $i=0$ is proved in [28]. By Corollary 6.4 and Lemma 6.5, the general $K 3$ surface in $\mathcal{F}^{\mathfrak{G}_{i}}$ corresponds uniquely to a five-dimensional projective space intersecting the Grassmannian $G\left(2, V_{6}\right) \subset \mathbb{P}^{14}$ in exactly $i$ points modulo automorphisms of $V_{6}$. Such $i$ secant 5-planes are unirationally parametrized by the product of the $i$-th symmetric product of $G\left(2, V_{6}\right)$ and $(6-i)$-th symmetric product of $\mathbb{P}^{14}$.

We remark that the unirationality of $\mathcal{F}^{\mathfrak{G}_{1}}$ can also be shown using quartic surfaces in $\mathbb{P}^{3}$ containing an elliptic quintic curve. The question of (uni)rationality of $\mathcal{F}^{\mathfrak{G}_{7}}$ and $\mathcal{F}^{\mathfrak{G}_{8}}$ is open.

\subsection{The moduli map}

Let $\mathcal{F}_{8}$ denote the 19-dimensional moduli space of polarized $K 3$ surface of genus 8 and $\mathcal{P}_{8}$ the moduli space of triples $(S, L, C)$ where $(S, L) \in \mathcal{F}_{8}$ and $C \in|L|$ is a smooth irreducible curve. Let $m_{8}: \mathcal{P}_{8} \longrightarrow \mathcal{M}_{8}$ be the moduli map.

Proposition 6.9 Let $(S, L) \in \mathcal{F}_{8}$ be a Brill-Noether general $K 3$ surface such that $S$ contains an elliptic pencil $|E|$ satisfying $E . L=5$. Then the fiber of $m_{8}$ is smooth and 6-dimensional at any point represented by a smooth curve $C$ in $|L|$.

Proof By comparing dimensions, the fibers of $m_{8}$ are at least 6-dimensional. (It is known that $m_{8}$ is dominant, and therefore its general fibers are precisely 6-dimensional, but we will not use this.) By [33, §3.4.4] or [8], the kernel of the differential of $m_{8}$ at a point $(S, L, C)$ is isomorphic to $H^{1}\left(\mathcal{T}_{S}(-L)\right)$. To prove the proposition, it therefore suffices by Serre duality to prove that $h^{1}\left(\Omega_{S}(L)\right) \leq 6$.

Let $\varphi: S \rightarrow \mathbb{P}^{3}$ be the morphism defined by $|L-E|$ and $S_{0}$ be its image, which is a quartic surface. By Lemma 6.2 its possible singularities are images of contracted disjoint rational curves $\Gamma_{i}$ on $S, i=1, \ldots, k$. By [25, Thm. 2.1] we have a short exact sequence

$$
0 \longrightarrow \mathcal{O}_{\Gamma_{1}+\cdots+\Gamma_{k}} \longrightarrow \varphi^{*} \Omega_{S_{0}} \longrightarrow \Omega_{S} \longrightarrow \mathcal{O}_{\Gamma_{1}+\cdots+\Gamma_{k}} \longrightarrow 0 .
$$

Twisting by $\mathcal{O}_{S}(L)$, taking cohomology and using the fact that $\Gamma_{i} \cdot L=1$ by Lemma 6.2 , we obtain

$$
h^{1}\left(\Omega_{S}(L)\right) \leq h^{1}\left(\varphi^{*} \Omega_{S_{0}}(L)\right)
$$


Pulling back the conormal bundle sequence

$$
\mathcal{O}_{S_{0}}(-4) \cong \mathcal{I}_{S_{0} / \mathbb{P}^{3}} /\left.\mathcal{I}_{S_{0} / \mathbb{P}^{3}}^{2} \longrightarrow \Omega_{\mathbb{P}^{3}}\right|_{S_{0}} \longrightarrow \Omega_{S_{0}} \longrightarrow 0
$$

and twisting by $\mathcal{O}_{S}(L)$, we obtain

$$
\left.\mathcal{O}_{S}(-3 L+4 E) \longrightarrow \varphi^{*} \Omega_{\mathbb{P}^{3}}\right|_{S_{0}}(L) \longrightarrow \varphi^{*} \Omega_{S_{0}}(L) \longrightarrow 0 .
$$

The left hand map is injective, as $\mathcal{O}_{S}(-3 L+4 E)$ is locally free. Thus,

$$
h^{1}\left(\varphi^{*} \Omega_{S_{0}}(L)\right) \leq h^{1}\left(\left.\varphi^{*} \Omega_{\mathbb{P}^{3}}\right|_{S_{0}}(L)\right)+h^{0}(3 L-4 E),
$$

using Serre duality. Pulling back the dual of the Euler sequence,

$$
\left.0 \longrightarrow \Omega_{\mathbb{P}^{3}}\right|_{S_{0}} \longrightarrow H^{0}\left(\mathcal{O}_{S_{0}}(1)\right) \otimes \mathcal{O}_{S_{0}}(-1) \longrightarrow \mathcal{O}_{S_{0}} \longrightarrow 0
$$

and twisting by $\mathcal{O}_{S}(L)$, we obtain

$$
\left.0 \longrightarrow \varphi^{*} \Omega_{\mathbb{P}^{3}}\right|_{S_{0}}(L) \longrightarrow H^{0}(L-E) \otimes \mathcal{O}_{S}(E) \longrightarrow \mathcal{O}_{S}(L) \longrightarrow 0 .
$$

Hence, since $h^{1}(E)=0$ as $E$ is irreducible, we obtain

$$
h^{1}\left(\left.\varphi^{*} \Omega_{\mathbb{P}^{3}}\right|_{S_{0}}(L)\right) \leq \operatorname{cork} \mu,
$$

where $\mu$ is the multiplication map of sections

$$
\mu: H^{0}(L-E) \otimes H^{0}(E) \longrightarrow H^{0}(L) .
$$

Combining (6.4), (6.5) and (6.6), we see that we obtain the desired inequality $h^{1}\left(\Omega_{S}(L)\right) \leq 6$ if we prove that

$$
h^{0}(3 L-4 E)=5
$$

and

$$
\text { cork } \mu=1 \text {. }
$$

To prove (6.8), note that the evaluation map $H^{0}(E) \otimes \mathcal{O}_{S} \rightarrow \mathcal{O}_{S}(E)$ is surjective as $|E|$ is base point free and has kernel $\mathcal{O}_{S}(-E)$. Twisting by $\mathcal{O}_{S}(L-E)$, we obtain

$$
0 \longrightarrow \mathcal{O}_{S}(L-2 E) \longrightarrow H^{0}(E) \otimes \mathcal{O}_{S}(L-E) \longrightarrow \mathcal{O}_{S}(L) \longrightarrow 0
$$

Taking cohomology and using the fact that $h^{1}(L-E)=0$ as $L-E$ is big and nef by Lemma 6.2, we obtain that cork $\mu=h^{1}(L-2 E)$.

We have $(L-2 E) . L=4$, hence $h^{2}(L-2 E)=h^{0}(2 E-L)=0$, as $L$ is ample. Similarly, $h^{0}(L-2 E)=0$, since $(L-2 E) .(L-E)=-1$ and $L-E$ is nef. Since $(L-2 E)^{2}=-6$, Riemann-Roch yields $h^{1}(L-2 E)=1$, and (6.8) is proved.

To prove (6.7), note that $(3 L-4 E)^{2}=6$ and $h^{2}(3 L-4 E)=h^{0}(4 E-3 L)=0$, as $(4 E-3 L) . E<0$ and $E$ is nef. Hence, (6.7) is equivalent to $h^{1}(3 L-4 E)=0$.

To get a contradiction, assume that $h^{1}(3 L-4 E)>0$. Then, by [19], there exists an effective divisor $\Delta$ such that $\Delta^{2}=-2$ and $k:=-\Delta .(3 L-4 E) \geq 2$. Since $\Delta . L>0$, as $L$ is ample, we must have

$$
\Delta . E \geq 2 .
$$


One computes $(3 L-4 E-k \Delta)^{2}=6$ and $(3 L-4 E-k \Delta) .(L-E)=7-k \Delta .(L-E)$. By the Hodge index theorem,

$$
24=(3 L-4 E-k \Delta)^{2} \cdot(L-E)^{2} \leq[7-k \Delta \cdot(L-E)]^{2},
$$

and the only possibilities are

(I) $\Delta .(L-E)=0$; or

(II) $\Delta .(L-E)=1$ and $k=2$.

In case (I) we find $(L-E-\Delta)^{2}=2$ and $(L-E-\Delta)$. $(L-E)=4$, whence $h^{0}(L-$ $E-\Delta) \geq 3$ by Riemann-Roch and Serre duality. By (6.9) we have $(E+\Delta)^{2} \geq 2$, thus also $h^{0}(E+\Delta) \geq 3$ by Riemann-Roch. But then $h^{0}(L-E-\Delta) h^{0}(E+\Delta) \geq 9=8+1$, contradicting Brill-Noether generality.

In case (II) we have $\Delta . L=\Delta . E+1$ and $-2=\Delta .(3 L-4 E)$, which together yield $\Delta . E=5$ and $\Delta . L=6$. Therefore, $(L-E-\Delta)^{2}=0$ and $(L-E-\Delta) . L=3$, it follows $h^{0}(L-E-\Delta) \geq 2$ by Riemann-Roch and Serre duality. Moreover, $(E+\Delta)^{2}=8$, whence $h^{0}(E+\Delta) \geq 6$ by Riemann-Roch. Similarly to the previous case, we obtain a contradiction to Brill-Noether generality.

This shows that (6.7) holds and finishes the proof of the proposition.

For $i \in\{0, \ldots, 9\}$, let $\mathfrak{G}_{i}$ and $\mathcal{F}^{\mathfrak{G}_{i}}$ be as in the previous subsection and let $\mathcal{P}^{\mathfrak{G}_{i}}$ be the moduli space of triples as in Sect. 2. Note that $\mathcal{P}^{\mathfrak{G}_{i}}$ is birational to the open part of the tautological $\mathbb{P}^{8}$-bundle over $\mathcal{F}^{\mathfrak{G}_{i}}$ consisting of pairs $(S, C)$ with $[S] \in \mathcal{F}^{\mathfrak{G}_{i}}$ and $[C]$ representing a smooth curve in $|L|$, where $L$ is the generator class of square 14 in $\mathfrak{G}_{i}$. We have $\mathcal{P}^{\mathfrak{G}_{i+1}} \subset \mathcal{P}^{\mathfrak{G}_{i}}$ for each $i \in\{0, \ldots, 8\}$.

Let $m_{8}^{\mathfrak{G}_{i}}: \mathcal{P}^{\mathfrak{G}_{i}} \rightarrow \mathcal{M}_{8}$ be the moduli map.

Proposition 6.10 For each $i \in\{0, \ldots, 9\}$, a general fiber of $m_{8}^{\mathfrak{G}_{i}}$ has dimension $\max \{0,6-i\}$.

Proof By Proposition 6.9, the fiber of $m_{8}^{\mathfrak{G}_{0}}$ is smooth and 6-dimensional at any point $(S, C) \in$ $\mathcal{P}^{\mathfrak{G}_{9}}$. Fix such an $(S, C)$.

We will show that there exists a chain of irreducible components $F_{i} \subset\left(m_{8}^{\mathfrak{G}_{i}}\right)^{-1}([C])$ of the fiber of $m_{8}^{\mathfrak{G}_{i}}$ for $i \in\{0, \ldots, 5\}$, respectively, containing $(S, C) \in \mathcal{P}^{\mathfrak{G}_{9}}$ such that

$$
(S, C) \in F_{5} \subsetneq F_{4} \subsetneq \cdots \subsetneq F_{1} \subsetneq F_{0} .
$$

Consequently, there exist $K 3$ surfaces $S_{i} \in \mathcal{F}^{\mathfrak{G}_{i}} \backslash \mathcal{F}^{\mathfrak{G}_{i+1}}$ for $i \in\{0, \ldots, 5\}$ containing $C$. Since $\operatorname{dim} F_{0}=6$ by Proposition 6.9, the dimension of $F_{i}$ is $6-i$ for $i \in\{0, \ldots, 5\}$ and the proposition will follow.

By construction, $S$ (resp. $C$ ) is the intersection of $G\left(V_{6}, 2\right)$ with a $\mathbb{P}^{8}$ (respectively a $\mathbb{P}^{7}$ ) in $\mathbb{P}^{14}$. The dual $\mathbb{P}^{5}$ of the $\mathbb{P}^{8}$, which we henceforth call $\mathbb{P}_{(S)}^{5}$, intersects the dual $G\left(2, V_{6}\right)$ in 9 points, call them $x_{1}, \ldots, x_{9}$, and the dual $\mathbb{P}^{6}$ of the $\mathbb{P}^{7}$, which we henceforth call $\mathbb{P}_{(C)}^{6}$, contains $\mathbb{P}_{(S)}^{5}$.

By construction, the nine points $x_{1}, \ldots, x_{9}$ span $\mathbb{P}_{(S)}^{5}$. Thus, we may find inside $\mathbb{P}_{(C)}^{6}$ a set of six additional hyperplanes $\mathbb{P}_{(i)}^{5}, i \in\{0, \ldots, 5\}$ containing precisely $i$ of the points $x_{1}, \ldots, x_{9}$; in particular $\mathbb{P}_{(i)}^{5}$ intersects $G\left(2, V_{6}\right)$ in precisely $i$ points.

Denote by $\mathbb{P}_{(i)}^{8}$ the dual $\mathbb{P}^{8}$ of $\mathbb{P}_{(i)}^{5}$. Then $\mathbb{P}_{(i)}^{8} \cap G\left(V_{6}, 2\right)$ is a $K 3$ surface $S_{i}$ containing $C$ and precisely $i$ elliptic pencils of degree 5 (and mutually intersecting in 2 points) by Corollary 6.4. As the nine elliptic pencils together with $C$ generate $\mathfrak{G}_{9} \subset \operatorname{Pic}(S)$, we also have that $C$ 
and the $i$ elliptic pencils generate $\mathfrak{G}_{i} \subset \operatorname{Pic}\left(S_{i}\right)$, whence $S_{i} \in \mathcal{F}^{\mathfrak{G}_{i}} \backslash \mathcal{F}^{\mathfrak{G}_{i+1}}$. Each pair $\left(S_{i}, C\right)$ therefore lies in $F_{i} \backslash F_{i+1}$. This concludes the proof.

Corollary 6.11 For each $i \in\{0, \ldots, 9\}$, the codimension of the image of the moduli map $m_{8}^{\mathfrak{G}_{i}}$ is $\max \{0, i-6\}$. In particular, a general curve of genus 8 is a linear section of a K3 surface such that precisely six out of its $14 g_{5}^{1}$ s are induced by elliptic pencils on the K3 surface. Moreover, there is a codimension $k$ family of curves lying on a $K 3$ surface such that precisely $6+k$ of its $g_{5}^{1}$ s are induced by elliptic pencils on the $K 3$ surface for $k \in\{1,2,3\}$.

Remark 6.12 One can ask similar questions for $K 3$ surfaces of higher even genus. For instance, how many elliptic pencils of minimal degree exist on a Brill-Noether general $K 3$ surface? But the methods in this article cannot be applied to $K 3$ surfaces of higher genus. Indeed, let $C$ be a Brill-Noether general curve of even genus $g \geq 10$. Note on the one hand that the curve $C$ does not lie on a $K 3$ surface and on the other hand that the (finite) number of pencils of minimal degree on $C$ is bigger that 19 (the maximal rank of the Picard lattice of a smooth $K 3$ surface). Furthermore, a characterization of Brill-Noether general $K 3$ surfaces is only known for $g \leq 10$ and 12 .

Acknowledgements The authors benefitted from conversations with Christian Bopp and Frank-Olaf Schreyer and acknowledge support from Grant No. 261756 of the Research Council of Norway.

Funding Open Access funding provided by Projekt DEAL.

Open Access This article is licensed under a Creative Commons Attribution 4.0 International License, which permits use, sharing, adaptation, distribution and reproduction in any medium or format, as long as you give appropriate credit to the original author(s) and the source, provide a link to the Creative Commons licence, and indicate if changes were made. The images or other third party material in this article are included in the article's Creative Commons licence, unless indicated otherwise in a credit line to the material. If material is not included in the article's Creative Commons licence and your intended use is not permitted by statutory regulation or exceeds the permitted use, you will need to obtain permission directly from the copyright holder. To view a copy of this licence, visit http://creativecommons.org/licenses/by/4.0/.

\section{References}

1. Aprodu, M., Farkas, G., Ortega, A.: Restricted Lazarsfeld-Mukai bundles and canonical curves. In Development of moduli theory-Kyoto 2013, Adv. Stud. Pure Math., vol. 69, pages 303-322. Math. Soc. Japan, [Tokyo] (2016)

2. Aprodu, M.: Lazarsfeld-Mukai bundles and applications. In Commutative algebra, pp. 1-23. Springer, New York (2013)

3. Arbarello, E., Cornalba, M.: Footnotes to a paper of Beniamino Segre, The number of $g_{d}^{1}$ 's on a general $d$-gonal curve, and the unirationality of the Hurwitz spaces of 4-gonal and 5-gonal curves. Math. Ann. 256(3), 341-362 (1981)

4. Arbarello, E., Cornalba, M., Griffiths, P.A., Joseph, Harris: Geometry of algebraic curves Vol I, Grundlehren der Mathematischen Wissenschaften, vol. 267. Springer, New York (1985)

5. Artebani, M., Kondō, S.: The moduli of curves of genus six and $K 3$ surfaces. Trans. Am. Math. Soc. 363(3), 1445-1462 (2011)

6. Artebani, M., Sarti, A.: Non-symplectic automorphisms of order 3 on $K 3$ surfaces. Math. Ann. 342(4), 903-921 (2008)

7. Barth, W. P., Hulek, K., Peters, C.A.M., Van de Ven, A.: Compact complex surfaces, Ergebnisse der Mathematik und ihrer Grenzgebiete. 3. Folge, vol. 4. Springer, Berlin, second edition (2004)

8. Beauville, A.: Fano threefolds and $K 3$ surfaces. In The Fano Conference, pp. 175-184. University of Torino, Turin (2004)

9. Bhargava, M., Ho, W., Kumar, A.: Non-symplectic automorphisms of order 3 on $K 3$ surfaces. Forum Math. Sigma 4:e18, 86 (2016) 
10. Ciliberto, C., Pareschi, G.: Pencils of minimal degree on curves on a $K 3$ surface. J. Reine Angew. Math. 460, 15-36 (1995)

11. Dolgachev, I.V.: Mirror symmetry for lattice polarized K3 surfaces. J. Math. Sci. 81(3), 2599-2630 (1996). Algebraic geometry, 4

12. Grayson, D.R., Stillman, M.E.: Macaulay2, a software system for research in algebraic geometry. http:// www.math.uiuc.edu/Macaulay2

13. Green, M., Lazarsfeld, R.: Special divisors on curves on a $K 3$ surface. Invent. Math. 89(2), 357-370 (1987)

14. Greer, F., Li, Z., Tian, Z.: Picard groups on moduli of K3 surfaces with Mukai models. Int. Math. Res. Not. IMRN 16, 7238-7257 (2015)

15. Hoff, M., Knutsen, A.L.: ancillary Macaulay2 file. https://www.math.uni-sb.de/ag/schreyer/index.php/ people/researchers/74-michael-hahn (2020)

16. Iskovskikh, V.A., Prokhorov, Y. G.: Fano varieties. In Algebraic geometry, V, Encyclopaedia Math. Sci., vol. 47, pp. 1-247. Springer, Berlin (1999)

17. Johnsen, T., Knutsen, A.L.: K3 projective models in scrolls. Lecture Notes in Mathematics, vol. 1842. Springer, Berlin (2004)

18. Knutsen, A.L.: On two conjectures for curves on $K 3$ surfaces. Internat. J. Math. 20(12), 1547-1560 (2009)

19. Knutsen, A.L., Lopez, A.F.: A sharp vanishing theorem for line bundles on $K 3$ or Enriques surfaces. Proc. Am. Math. Soc. 135(11), 3495-3498 (2007)

20. Kodaira, K.: On the structure of compact complex analytic surfaces I. Am. J. Math. 86, 751-798 (1964)

21. Kondō, S.: The moduli space of curves of genus 4 and Deligne-Mostow's complex reflection groups. In Algebraic geometry 2000, Azumino (Hotaka), Adv. Stud. Pure Math., vol. 36, pp. 383-400. Math. Soc. Japan, Tokyo (2002)

22. Lazarsfeld, R.: Brill-Noether-Petri without degenerations. J. Differ. Geom. 23(3), 299-307 (1986)

23. Lelli-Chiesa, M.: Stability of rank-3 Lazarsfeld-Mukai bundles on K3 surfaces. Proc. Lond. Math. Soc. (3) 107(2), 451-479 (2013)

24. Morrison, D.R.: On K3 surfaces with large Picard number. Invent. Math. 75(1), 105-121 (1984)

25. Morrison, D.R.: The birational geometry of surfaces with rational double points. Math. Ann. 271(3), 415-438 (1985)

26. Mukai, S.: Curves and K3 surfaces of genus eleven. In Moduli of vector bundles (Sanda, 1994; Kyoto, 1994), volume 179 of Lecture Notes in Pure and Appl. Math., pp. 189-197. Dekker, New York (1996)

27. Mukai, S.: Curves and Grassmannians. In Algebraic geometry and related topics (Inchon, 1992), Conf. Proc. Lecture Notes Algebraic Geom., I, pp. 19-40. Int. Press, Cambridge, MA (1993)

28. Mukai, S.: Curves, $K 3$ surfaces and Fano 3-folds of genus $\leq 10$. In Algebraic geometry and commutative algebra, Vol. I, pp. 357-377. Kinokuniya, Tokyo (1988)

29. Mukai, S.: New developments in the theory of Fano threefolds: vector bundle method and moduli problems [translation of Sūgaku 47 (1995), no. 2, 125-144; MR1364825 (96m:14059)]. Sugaku Expositions, 15(2):125-150, (2002). Sugaku expositions

30. Mukai, S.: Biregular classification of Fano 3-folds and Fano manifolds of coindex 3. Proc. Nat. Acad. Sci. USA 86(9), 3000-3002 (1989)

31. Nikulin, V.V.: Biregular classification of Fano 3-folds and Fano manifolds of coindex 3. Izv. Akad. Nauk SSSR Ser. Mat 43(1), 111-177, 238 (1979)

32. Saint-Donat, B.: Projective models of $K-3$ surfaces. Am. J. Math. 96, 602-639 (1974)

33. Sernesi, E.: Deformations of algebraic schemes, Grundlehren der Mathematischen Wissenschaften, vol. 334. Springer, Berlin (2006)

34. Shepherd-Barron, N.I.: Invariant theory for $S_{5}$ and the rationality of $M_{6}$. Compos. Math. 70(1), 13-25 (1989)

Publisher's Note Springer Nature remains neutral with regard to jurisdictional claims in published maps and institutional affiliations. 